\title{
TV-kiosk: An open and extensible platform for the wellbeing of an ageing population
}

\author{
Maarten Steenhuyse, Jeroen Hoebeke, Ann Ackaert, Ingrid Moerman, Piet Demeester \\ Ghent University - IBBT, Department of Information Technology (INTEC), \\ Gaston Crommenlaan 8 Bus 201, 9050 Ghent, Belgium \\ \{firstname.lastname\} eintec.ugent.be
}

\begin{abstract}
The ageing population is becoming a growing challenge to society. User-centered ICT solutions adapted to elderly can play a prime role in dealing with these challenges. This paper presents TV-kiosk, an open and extensible TV-based platform that aims to stimulate social interaction, avoid isolation and deliver information. One of its distinguishing features is the underlying decentralized network communication approach based on advanced technology to securely and automatically interconnect devices and the TV-based user interface specifically designed for elderly. The openness and extensibility of the platform make it possible to easily integrate all kinds of new and existing eservices. Currently, a field trial is ongoing in a care center where TV-kiosk is being used by elderly, their family and caregivers.
\end{abstract}

Keywords: Elderly people, e-services, well-being, TV, platform, network virtualization, decentralized

\section{Introduction}

ICT is continuously evolving at a very fast pace and plays an ever increasing role in our daily life. Technologies such as the Internet, mobile phones, social networks, interactive digital TV, etc. have become an indispensable part of our life, but not of everybody's life. Despite their popularity, these ICT technologies have not yet strongly penetrated into the daily life of many elderly. The majority of them is not computer literate, which make them digital outsiders for the current web applications and digital devices that are frequently used by their relatives.

This observation conflicts with the fact that innovation can enhance their quality of life, can help to cut the high costs that come with the ageing population and can create new business opportunities. Although there is a clear need, large opportunities and important role for ICT technologies to support the life of these people and to improve society, the reality is still different. However, the last few years, a lot of initiatives are trying to bridge this gap by designing ICT solutions specifically tailored to elderly and applying multi-disciplinary design methodologies in order to increase the accessibility and acceptance by the target group.

In this paper, we present such an initiative, TV-kiosk, which is the result of several years of cross-disciplinary research and which aims to stimulate social interaction, 
avoid isolation and deliver information in a way adapted to elderly. The paper is structured as follows. In section 2, we describe in more detail the challenges we want to address and the requirements and assumptions we have put forward for the design of our platform. Section 3 describes the advanced networking technology behind the platform, whereas section 4 gives an overview of the services that have been designed and deployed. In section 5, we briefly describe the ongoing field trial of TV-kiosk in a care center, where the platform is being used by our target group, their family and caregivers. An analysis of the platform and comparison with related work in this field is subject of sections 6 and 7. Finally, section 8 concludes this paper.

\section{Problem statement and approach}

The ageing population is becoming a growing challenge to society in the industrialized world. By 2020, a quarter of Europe's population will be aged over 65 years. By 2050, people aged between 65 and 79 will make up almost a third. At the same time, the pool of potential caregivers is shrinking. New ICT technologies can help to meet the challenges of an ageing population in several areas. They can help them to carry out daily activities, to stimulate their social participation, to monitor their health, to facilitate social inclusion, to avoid isolation and loneliness or to enhance independent living.

In practice, the majority of elderly people does not yet enjoy the benefits of digitalization for several reasons. There is the age-related decline in cognitive functions, which has a profound effect on the capabilities of elderly in using new technologies [1]. The current interface design does not match these cognitive capabilities, and is sometimes even difficult for technology-prone people [2]. Last but not least, there is the overall acceptance of technologies based on issues such as control, trust, privacy, dignity or usability [3]. As such, designing new technologies is a problem with many facets, requiring multi-disciplinary efforts crossing the entire development spectrum and involving all stakeholders [4].

In this paper, we will focus on two important areas where ICT can make an important contribution: social inclusion and information access. Elderly are vulnerable to social isolation and have less access to information. Family and friends often have a busy life or live at distant locations, not having time for regular visits. However, social interaction with family and friends is important for their wellbeing and health. Within a care setting, caregivers spent a lot of time on administrative tasks, already reducing the time they can spend on their core task, providing care to people. With the decrease of workforce in the healthcare sector, this problem will become even more prevalent. However, access to information about the world and the environment they live (e.g. care center) in is important, offering a window to the world and helping to feel connected with society.

For these areas we wanted to realize a user centered ICT platform, usable by elderly people not acquainted with ICT technologies at all. At the same time, it had to address the above issues related to the acceptance and accessibility of new ICT technologies by following a multi-disciplinary design methodology. Next to this, we outlined a number of other requirements and assumptions that have driven the design of the platform. If we look at a Flemish study on the use of digital media by elderly, 
we can observe that TV is most popular [5]. This suggests that TV is an attractive medium to provide ICT solutions usable by the target group with little training. As such, the TV set was chosen as a primary interaction screen for the elderly, a PC or laptop for the other involved actors. To interconnect all actors securely over the Internet and to share and discover services, a secure, flexible and self-organizing solution was needed, hiding all underlying technological complexity. For the platform, we had several services in mind within our two target areas, but we wanted the platform to be extensible and open as well. Extensible, in order to be able to include other services - addressing other areas relevant to an ageing population - that could be deployed according to evolving needs of the users or in order to be able to integrate existing Internet-based services within the simplified user interface. Open, in order to allow other parties to design new services for the platform, which could lead to new and innovative services and accelerate the uptake of the solution.

Based on the above requirements and assumptions, the TV-kiosk platform was built, which will be further presented in the following sections.

\section{The technology}

As stated, we require a secure, flexible and self-organizing solution in order to interconnect all actors (e.g. elderly, family, caregivers) over the Internet. In order to have an open and extensible service platform, we have chosen not to adopt a centralized solution controlled by a single service provider as is mostly being done. Instead, we have used our Virtual Private Ad-Hoc Network (VPAN) platform which combines ad hoc networking, peer-to-peer techniques and ubiquitous computing aspects to realize communication between trusted groups of devices [6].

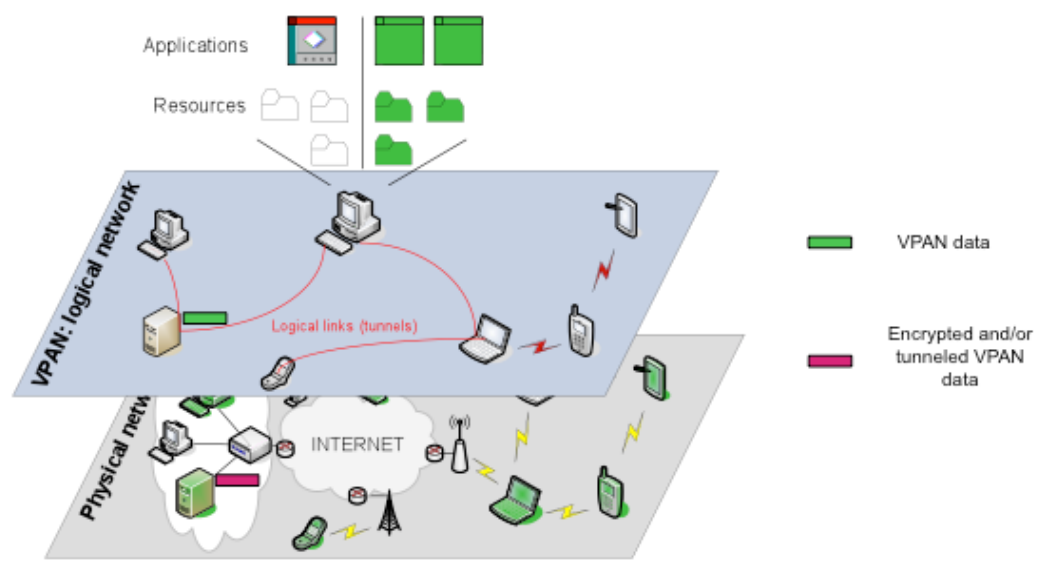

Fig. 1. Virtual Private Ad-Hoc Network concept

Through this VPAN technology, multiple devices at distant locations that need secure and easy access to each other's data and services are fully automatically and securely grouped in a logical, virtual network, regardless of the heterogeneity of underlying networks. Based on security information, devices can verify if they are 
member of the same group, after which they can setup secure connections to exchange information. The virtual network will organize and maintain itself, regardless of the devices' location, temporary interruptions in connectivity or upon mobility. No user interaction is required. The technology also contains a service framework that automatically announces the availability of shared services to other members of the same group and allows user-friendly access and management. Any service can be deployed on top of the logical network. For more details on the VPAN platform we refer to [7].

As such, the VPAN technology effectively hides the technological complexity of the interconnection between devices and is able to create permanent and closed groups. Furthermore, the technology meets the flexibility requirements, not only in terms of devices and services, but a device can also be member of multiple groups, which are all nicely shielded from each other.

Figure 2 illustrates how the VPAN technology is now being used in the TV-Kiosk platform. Secure and separated VPANs allow users to easily interact with each other. The "Home for the Elderly" VPAN offers services related to the care center. All residents are member of this group. On the other hand, every resident can be member of a private (for his family) "Family" VPAN, which allows family members to offer services such as photo sharing from their home.

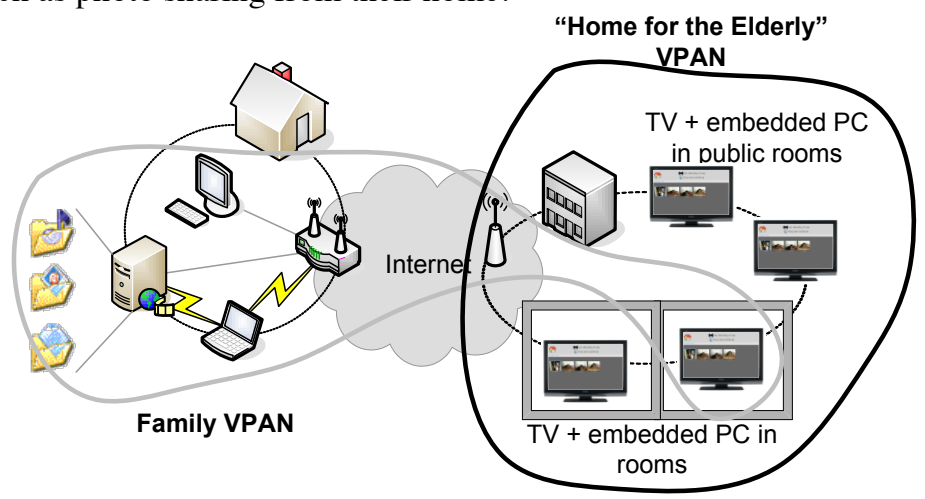

Fig. 2. Family and elderly home VPANs

\section{The services}

On top of the networking and service discovery functionality offered by VPAN, we developed an accessible user interface for elderly on their TV. With a simple remote control users can access the service of choice in a few steps. To keep navigation simple, the remote has as few buttons as possible; a home button enables them to return to the start screen at any time. The start screen corresponds with the groups (i.e. VPANs) the user is member of.

Figure 3 depicts schematically where the content can originate from in our prototype setup. Next to content delivered using VPAN networks it is also possible to access Internet services on condition these are simple, preferably tailored with the target group in mind, and being integrated in the user interface. To enable 
extensibility, a plugin system has been used to implement the services and easily allow the creation of new services. In the following paragraphs we elaborate further on the design requirements we considered and the developed services.

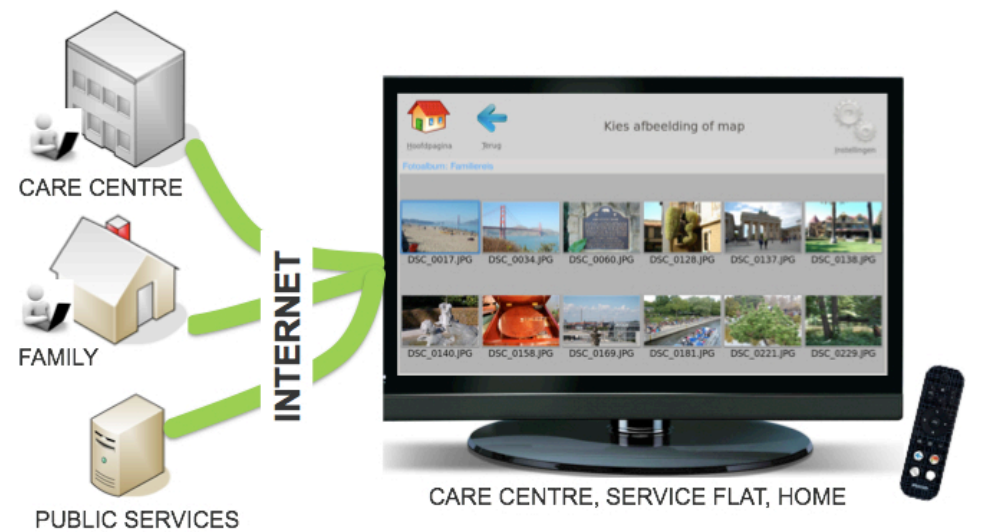

Fig. 3. The TV-Kiosk prototype

As mentioned before, in order to reach the target group, technological complexity must be hidden as much as possible. To this end, we collaborated closely with usability experts, people in the field such as care providers and last but not least the targeted end users. Development took place in different phases, collecting feedback obtained through usability research and setting up field trials to improve the product and obtain a satisfactory final result. For the user interfaces, the following usability guidelines have been taken into account during design:

- User Interfaces should be as simple as possible. The more information a screen holds, the more likely the user gets confused. A better approach is to use more screens than to try to fit everything on one screen.

- User interaction should be as simple as possible. Interaction choices should be kept at a minimum. Regular keyboards and mouse are usually inappropriate for the targeted users.

- The user should be guided as much as possible. A tutorial like interface is needed where every possible step is clearly explained.

Figure 4 shows the TV-Kiosk platform and some services in action. The top left picture shows the TV setup with the photo viewer service and the top right shows the music player. The screenshots at the bottom show how information can be displayed to the user, both for a VPAN service (activities in the care center) and a public Internet service (weather forecast).

For the service providers, such as staff in the care center and family members, a PC version of the software is available with plugins to share and/or create content. Next to this, we also built a web application that enables elderly to access messages and pictures posted by a relative using a Facebook plugin. This nicely illustrates how widely used web services can be easily integrated into our platform and complement our services running on top of the decentralized VPAN platform. 


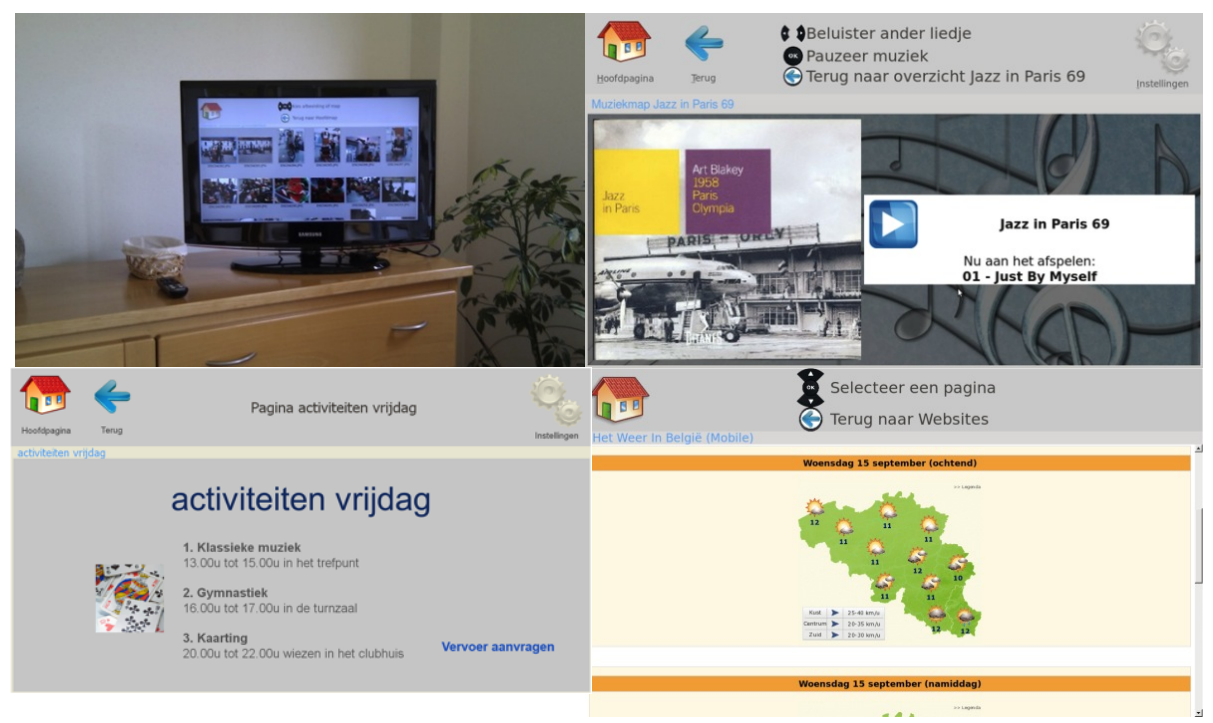

Fig. 4. Screenshots services

\section{The field trial}

We deployed the TV-Kiosk platform in a retirement home where three residents and family members were selected based on the mental fitness of the residents and the willingness to cooperate. We installed our system in the room of the residents and in a public room. We equipped a PC used by the staff with the PC version of our software. This PC acts as content provider for the services in the "Home for the Elderly" group, which maps to a VPAN on the networking level. Typical services involve information about the retirement home: planned activities, menu of the week, pictures of past activities, etc. The family members also installed the PC version of the software on their computer at home. This allowed them to create a "Family" group, enabling them to share pictures, music, messages and other content. Finally, a third group "External" is available which can be used for third-party providers that have an agreement with the retirement home to provide content. For example, a museum can provide pictures of the latest exhibitions.

During an initial field trial, TV-kiosk was tested, feedback collected and improvements were made. Currently a second field trial is ongoing using the enhanced version of our platform [8]. From this first field trial we have learned that a major motivation to participate was that the platform enables the residents and their relatives to have regular contact without the need to move to the retirement home. Despite the user-friendly television interface, some residents still have some difficulty using it and need assistance from staff or family. Furthermore, even family members sometimes lack the basic computer knowledge to manage the sharing of pictures and other content despite the user-friendly interface. Therefore, it is important to provide clear manuals and support. Also, the residents typically see a television as a passive 
medium. They have to get used to the active input required to access the information they want. Furthermore, they experience television as a broadcast medium and do not always realize that they have their own private network (group) with their family. This must be clearly communicated to the residents.

\section{Requirement analysis}

Throughout the design and implementation of the TV-kiosk platform we have ensured to meet all the requirements we have put forward in the beginning. In cooperation with usability experts, we applied a user-centered multi-disciplinary design methodology consisting of the following steps. In a first step we analyzed the needs of the target group, developing a first concept. Secondly, the concept was translated into technological solutions - networking, services and user interfaces -, accompanied by technology assessment evaluations. This resulted in a third - ongoing - step were TVkiosk has been deployed and is being evaluated both on technical and social level during two trials in a retirement home. This approach is key to realize acceptance and accessibility of the newly designed ICT solutions. For more details on the applied methodology, we refer to [9].

From a technological point of view, we have chosen to use the VPAN platform, allowing the creation of multiple secure and self-organizing virtual IP networks according to the users needs and on top of which any service can be deployed and run. Apart from a one-time configuration step, this technology succeeds in hiding all underlying complexity in securely interconnecting the devices used by the different actors and automatically discovering shared services.

By taking a decentralized communication approach, opposed to centralized solutions, we avoid central control over the services that can be deployed and over the data exchanged or stored. In combination with an API to make use of the service discovery framework and to integrate services in the user interface as new plugins, any developer is able to create its own applications that can run within the logical VPAN networks, forming the basis of an open and easily extendable platform. Finally, next to the deployment of services within these virtual networks, we have also demonstrated the integration of existing Internet-based services within our simplified user interface. As such, our platform is capable of supporting both services running within the shielded logical networks as well as existing web-based services, combining the best of both worlds.

The above discussion reveals how TV-kiosk is capable of meeting the requirements and has taken precautions to ensure the accessibility of the technology. From the ongoing field trial, we are continuously collecting feedback that helps us to further improve the platform.

\section{$7 \quad$ Related work}

There are some other efforts also targeted to elderly and aiming to avoid social isolation, to stimulate social interaction or to deliver information. In this section we will provide an overview of the most relevant work in these fields within Europe and 
will compare it with the approach taken by TV-kiosk. We will restrict ourselves to those initiatives that also make use of Internet for communication and of TV as the primary interaction screen, which is best suited for people not acquainted with ICT.

Within Europe there are several, both finished as well as ongoing, projects related to TV-kiosk, especially within the AAL Joint Programme. Table 1 gives an overview of the most important European initiatives.

Table 1. Related EU initiatives

\begin{tabular}{|c|c|}
\hline Name & Short description and main characteristics \\
\hline $\begin{array}{l}\text { T-Seniority } \\
{[10]}\end{array}$ & $\begin{array}{l}\text { The T-seniority project (Expanding the Benefits of Information Society to } \\
\text { Older People through Digital TV Channels) aims to build an online service } \\
\text { platform that offers e-Care Services primarily via digital and digital interactive } \\
\text { TV. A citizen or patient sitting in front of TV screen, with a remote control on } \\
\text { hand, will be able to choose among many different options of public or } \\
\text { personalized services. The services are deployed on the Internet using a } \\
\text { Software as a Service model and is aimed to integrate many different services. }\end{array}$ \\
\hline $\begin{array}{l}\text { ALICE } \\
{[11]}\end{array}$ & $\begin{array}{l}\text { The ongoing ALICE project (Advanced Lifestyle Improvement system \& new } \\
\text { Communication Experience) recognizes social engagement with relatives and } \\
\text { friends as an important determinant of quality of life. The overall objective is to } \\
\text { research, develop and integrate a set of ICT-based services into the existing TV } \\
\text { set allowing elderly people to enjoy experiences of communication and social } \\
\text { interaction based on ICT and simplified access to Web resources. The system } \\
\text { will consist of a set-top box connected to the TV and a remote control tailored } \\
\text { to elderly. Also, an intuitive user interface will be developed allowing simple } \\
\text { navigation and control. The final system should also offer services like personal } \\
\text { alarming systems or health monitoring devices. A pilot installation is foreseen. }\end{array}$ \\
\hline $\begin{array}{l}\text { FoSIBLE } \\
{[12]}\end{array}$ & $\begin{array}{l}\text { The ongoing FoSIBLE project (Fostering Social Interactions for a Better Life } \\
\text { of the Elderly) considers social interactions beyond the near environment with } \\
\text { remotely living family members and friends to be equally important as social } \\
\text { interaction within the neighborhood. Different applications will be developed } \\
\text { and integrated in a TV-based Social Media Center. Also, issues related to } \\
\text { usability, user acceptance and accessibility will be specifically addressed. }\end{array}$ \\
\hline $\begin{array}{l}\text { HOMEdot } \\
\text { OLD [13] }\end{array}$ & $\begin{array}{l}\text { The ongoing HOMEdotOLD project aims to provide a highly personalized, } \\
\text { intuitive and open TV-based platform to advance the social interaction of } \\
\text { elderly people, aiming to prevent isolation and loneliness. The architecture is } \\
\text { centralized consisting of a set-top box and an external application server where } \\
\text { the services reside. }\end{array}$ \\
\hline $\begin{array}{l}\text { Join-in } \\
{[14]}\end{array}$ & $\begin{array}{l}\text { The recently started Join-In project (Senior Citizens Overcoming Barriers by } \\
\text { Joining Fun Activities) aims at providing technologies for elderly persons to } \\
\text { participate in social activities such as games and exer-games and have fun via } \\
\text { digital media. The Internet can also be accessed via TV, an interface the entire } \\
\text { target group is well acquainted with, by offering set-top boxes. Design-based } \\
\text { research will be used. }\end{array}$ \\
\hline
\end{tabular}

From Table 1 it is clear that similar initiatives exists that aim to design TV-based solutions for elderly in order to stimulate social interaction and avoid isolation. In many cases, the research methodology also explicitly focuses on usability and accessibility and field trials or pilots are seen as a crucial part of this process. It should be noted that, apart from T-Seniority, all projects have only recently started, 
not having published concrete results or mentioning to have reached a field trial phase. In that respect, TV-kiosk can be seen as one of the first initiatives that has succeeded in building a TV-based platform and evaluating it in a field trial. Therefore, these other initiatives strengthen our belief that a TV-based platform is a good approach to bring ICT technologies to elderly in view of user acceptance and accessibility.

When considering the services that are being offered or targeted, it should be noticed that some of the projects foresee a plethora of services, going beyond services currently offered by TV-kiosk, which primarily focus on social inclusion or dissemination of information. However, through our open and extensible design, we are confident to include more health-care related services in the future.

When looking at the technology behind the solutions, it can be said that, based on the publicly available information on the mentioned projects, the TV-kiosk approach is unique by taking a decentralized communication approach based on an advanced networking technology to securely and automatically interconnect devices both locally and over the Internet. The other solutions are centralized, where the offered services reside on a server. It is our belief that our decentralized approach has some interesting advantages, especially in view of the creation of an open and extensible platform for which anyone should be able to design novel services. However, we also have the possibility to integrate existing Internet services or centralized services, combining the best of both worlds, depending on the type of service being considered.

\section{Conclusions and future work}

It is clear that there is a strong need for ICT technologies tailored to elderly and designed in a cross-disciplinary way, starting from design up to field trial evaluation, in order to increase acceptance and accessibility. Hereby the fact that the majority of elderly is not computer literate and that there is an age-related decline in cognitive functions, impacting the capabilities in using new technologies, should be taken into account. Currently, several initiatives have emerged in Europe that recognize these issues and that start building solutions using TV as the prime interaction screen, since this is the medium elderly are most familiar with and are able to use with little training.

The TV-kiosk platform presented in this paper is one such an initiative for an ageing population that aims to stimulate social interaction, avoid isolation and deliver information. One of its distinguishing features is the underlying decentralized network communication approach based on advanced technology to securely and automatically interconnect devices and the user interface specifically adapted to elderly. The openness and extensibility of the platform make it possible to easily integrate all kinds of new and existing e-services. Next to this, a second field trial is already taking place, whereas most other initiatives have only recently started in designing and building TV-based solutions targeted to elderly.

Although a lot of research has been carried out already in order to create the platform, there is still a lot of room for additional research and extensions. For example, since government is stimulating the independent living of elderly, it is interesting to broaden our scope and extend our field trial in order to target also 
elderly living independently. New and more interactive e-services will be developed for this case such as the ordering of meals or the arrangement of transport. Also the extension with more health-related services such as monitoring needs to be considered. Such a wide variety of services on top of an open and extensible platform should result in a customizable system that can be easily deployed and extended according to the evolving needs of elderly. The advent of small and low-power sensors makes it interesting to extend our networking technology and platform in order to be able to incorporate sensors as well. Finally, as part of the crossdisciplinary approach, also the business aspect should be investigated, looking at all involved stakeholders and organizations in control of healthcare financing. This, in combination with the technology presented here is required to make such a platform a reality and contribute to a better and smarter society for an ageing population.

\section{References}

1. Slegers, K., van Boxtel, M.P.J., Jolles, J.: The Efficiency of Using Everyday Technological Devices by Older Adults: The Role of Cognitive Functions. Aeging and Society 29(2), pp. 309--325 (2009)

2. Wilkowska, W., Ziefle, M., Arning, K.: Older Adults' Navigation Performance when Using Small-screen Devices: Does a Tutor Help? In: $9^{\text {th }}$ International Conference on Work With Computer Systems, Beijing, China (2009).

3. Pew, R., Van Hemel, S.: Technology for Adaptive Aging. National Academies Press, Atlanta (2004)

4. Slegers, K.: Research Approaches into Communication Technologies for Older Users. In: CHI - Workshop Age Matters: Bridging the Generation Gap through Technology-mediated Interaction, Boston (2009)

5. Verté, D., De Donder, L.: Schaakmat of aan Zet? Monitor voor Lokaal Ouderenbeleid in Vlaanderen. Vandenbroele (2007)

6. Hoebeke, J., Holderbeke, G., Moerman, I., Dhoedt, B., Demeester, P.: Virtual Private Ad Hoc Networking. Wireless Personal Communications 38(1), pp. 125-141 (2006)

7. Hoebeke, J.: Adaptive Ad Hoc Routing and its Application to Virtual Private Ad Hoc Networks. Ph.D. thesis, Ghent University (2007)

8. TV-kiosk Press Conference, http://www.smartcareplatform.eu/content/tv-kiosk-pressconference

9. Ackaert, A., Jacobs, A., Veys, A., Derboven, J., Van Gils, M., Buysse, H., Agten, S., Verhoeve, P.: A multi-disciplinary approach towards the design and development of value+ eHomeCare services. In: Handbook of Digital Homecare, pp. 243--267. Springer, Berlin, (2009)

10.Moumtzi, V., Farinos, J., Wills, C.: T-Seniority: an online service platform to assist independent living of elderly population. In: $2^{\text {nd }}$ International Conference on Pervasive Technologies Related to Assistive Environments, Corfu, Greece (2009)

11.Advanced Lifestyle Improvement System and New Communication Experience, http://www.aal-alice.eu

12.Fostering Social Interactions for a Better Life of the Elderly, http://fosible.eu

13.Home Services Advancing the Social Interaction of Elderly People, http://www.homedotold.eu

14.Senior Citizens Overcoming Barriers by Joining Fun Activities, http://www.itfunk.org/docs/prosjekter/AAL-Join-In.htm 